\title{
ESTUDIO DE TÉCNICAS DE MEJORA DE LA PRODUCCIÓN DE DOS SELECCIONES DE TOMATE VALENCIANO
}

\author{
J.M. Aguilar, A. Giner, C. Baixauli*, I. Nájera, A. Núñez \\ Centro de Experiencias de Cajamar en Paiporta. \\ Apartado 194. 46200 Paiporta, Valencia

\section{*carlosbaixauli@,cajamar.com}

\section{Resumen}

El tomate (Lycopersicon esculentum Mill.) y sus diferentes selecciones de valenciano, son muy apreciados por consumidores a nivel local, debido a sus buenas características organolépticas. Estas selecciones no han sido sometidas a procesos de mejora genética, siendo el agricultor por selección masal quién conserva la semilla. En esta experiencia se utilizaron 2 selecciones de tomate valenciano, en las que se comparó planta injertada sobre el cv Arnold y planta sin injertar, con sistema de cultivo sin suelo, para lo cual se compararon 2 volúmenes de fibra de coco: sacos de 18 litros y 28,8 litros compuestos de una mezcla de $70 \%$ de fibra de coco y $30 \%$ de chips de coco. La experiencia se desarrolló en ciclo de primavera en un multitúnel con cubierta de malla de $14 \times 10$ hilos de polietileno. El riego fue independiente para cada volumen de sustrato, con una dotación de riego que se calculó a partir de la hipótesis de nivel de agotamiento del $10 \%$, considerando drenaje del $30 \%$. La mayor producción comercial se obtuvo con el menor volumen de sustrato, sobre este se obtuvo un mayor destrío por podredumbre apical. La planta injertada produjo más que la planta sin injertar, con un mayor peso medio de los frutos, vigor de las plantas y menor destrío. Se observaron diferencias de comportamiento productivo entre selecciones, así como interacciones en la producción de destrío y vigor de las plantas.

Palabras clave: injerto, fibra de coco, drenaje, dotación de riego, volumen sustrato. 


\section{Abstract}

The tomato Valenciano is very appreciated by consumers, especially in the province of Valencia, due to its good organoleptic characteristics. These cultivars do not have resistance to pests and diseases. The farmer himself, after a mass selection, is who mainly develop the tasks of conservation and reproduction of the seeds. Two cultivars of tomato valenciano were used for the development of the experience and they were analyzed different crop management. On the one hand it was studied the behavior of the plant that it was grafted on the cv Arnold and it was compared with a control without grafting. On the other hand the experiment was carried out in a soilless system and they were compared 2 coconut fiber volumes: on the basis of 18 liters and 28.8 liters sacks, both of them composed of a mixture of $70 \%$ of coconut fiber and $30 \%$ of coconut chips. The experience was developed in the spring cycle in a multitunnel mesh cover of $14 \times 10$ threads of polyethylene. Irrigation was independent for each substrate volume. The watering regime was determined on the basis of the hypothesis of $10-30 \%$ depletion level from the drainage. The largest commercial production was obtained with the lower volume sack and there had also been an increase amount of non-commercial yield, as a result of fruits affected by blossom end rot. Grafted plants achieved a greater commercial production that plants without grafting. Moreover, those ones grafted reached also a higher average weight of the fruits and lower noncommercial production, mainly due to the elevated incidence of blossom end rot in plants without grafting. Grafted plants were more vigorous than those ones non- grafted. There were detected differences between cultivars for productive performance, as well as interactions for non-commercial production and plant vigor.

Keywords: grafting, coconut fiber, irrigation requirements.

\section{1.- INTRODUCCIÓN}

Los sistemas de cultivo sin suelo son una alternativa eficiente para el cultivo de hortalizas locales sensibles a plagas y enfermedades de suelo, como el tomate valenciano (Baixauli, C y Aguilar J.M., 2002). Reducir el volumen de sustrato puede ser una buena solución para minorar los costes de producción. Por otra parte el injerto es una alternativa ecológica que puede ser utilizado para el control de plagas y enfermedades de suelo, así como reducir problemas debidos a agentes abióticos como salinidad, sequía, 
temperatura, encharcamiento (Miguel et al., 2007). Se ha estudiado el comportamiento de portainjertos como los cvs. Arnold, Armstrong, King Kong, Optifort y Groundforce, que con tomate valenciano se constató que con cualquiera de ellos se mejora la producción, el vigor y se reduce el ataque de nematodos, frente a un tomate sin injertar, mejorando en general el comportamiento agronómico (Giner et al., 2014).

En esta experiencia utilizando 2 selecciones de tomate valenciano, se compara la respuesta del tomate injertado y sin injertar, así como el comportamiento de dos volúmenes distintos de fibra de coco en un sistema de cultivo sin suelo.

\section{2.- MATERIAL Y MÉTODOS}

Se utilizaron 2 selecciones de tomate valenciano, la del agricultor Juan Giner (Alboraya) y la de Vicente Peris (Pobla Vallbona). Se comparó planta injertada sobre el cv Arnold y sin injertar. El tomate se desarrolló en sistema de cultivo sin suelo, para lo cual se compararon 2 formatos de saco con fibra de coco nuevos de la firma Projar (goldengrow) de 18 y 28,8 litros con dimensiones (longitud, ancho y alto): $100 \times 18 \times 10 \mathrm{~cm}$ y $100 \times 18 \times 16 \mathrm{~cm}$ respectivamente, rellenos de una mezcla de $70 \%$ de fibra de coco y $30 \%$ de chips de coco. La siembra del tomate valenciano destinado a injerto se realizó el 30 de diciembre de 2013, el portainjerto el 3 de enero de 2014 y el tomate que no iba a ser injertado el 15 de enero. El injerto se realizó de púa por encima del cotiledón el 6 de febrero y la plantación tuvo lugar el 17 de marzo. Para ello se dispusieron 3 plantas por saco que se podaron a 2 tallos con una densidad de 1,43 plantas $\mathrm{m}^{-2}$. La experiencia se desarrolló bajo una estructura de invernadero multitúnel con cubierta de malla de 14x10 hilos de polietileno, en el Centro de Experiencias de Cajamar en Paiporta. Se realizó un diseño estadístico de bloques al azar con 3 repeticiones y 6 plantas por parcela elemental. El riego fue independiente para cada volumen de sustrato, estableciendo su dotación a partir de la hipótesis de nivel de agotamiento del $10 \%$ y un drenaje previsto del $30 \%$, para lo cual se establecieron riegos de 11 minutos para los sacos de 18 litros y de 15 minutos para los de 28,8 litros.

Se midió la producción comercial, el peso medio de esos frutos medido en todas las observaciones. Se contabilizó también la producción de destrío, clasificada por las diferentes causas. El 13 de junio se hizo una valoración del altura de las plantas y frondosidad utilizando un índice de 0 a 5 de menor a mayor. 
Los resultados fueron analizados mediante análisis de la varianza múltiple con separación de medias mediante el test LSD $(\mathrm{p} \leq 0.05)$ con el programa informático Statgraphics Plus.

\section{3.- RESULTADOS}

La recolección se inició el 28 de mayo de 2014 y el ensayo finalizó el 6 de agosto, se realizaron un total de 29 recolecciones. La mayor producción comercial final se obtuvo con el tomate cultivado sobre los sacos de 18 litros, observando diferencias significativas a nivel estadístico (d.s.n.e.) respecto el de 28,8 litros. Entre selecciones la más productiva fue la de Vicente Peris aunque no se apreciaron d.s.n.e. entre ellas. La mayor producción comercial final se obtuvo con las plantas injertadas observando d.s.n.e. respecto aquellas sin injertar. En relación al peso medio de los frutos, no se observaron d.s.n.e. entre volúmenes de sustrato. La selección de Vicente Peris presentó un mayor peso medio de sus frutos, detectando d.s.n.e. durante la producción de junio y julio, aunque no se apreciaron d.s.n.e. en la observación del mes de agosto. Los frutos procedentes de la planta injertada también dieron frutos de mayor peso medio, aunque en este caso sólo se detectaron d.s.n.e. en las observaciones correspondientes a los meses de junio y agosto. No se observaron interacciones e.s. para el rendimiento comercial ni peso medio de sus frutos.

Tabla 1. Datos productivos. Rendimiento comercial acumulado y peso medio de los frutos

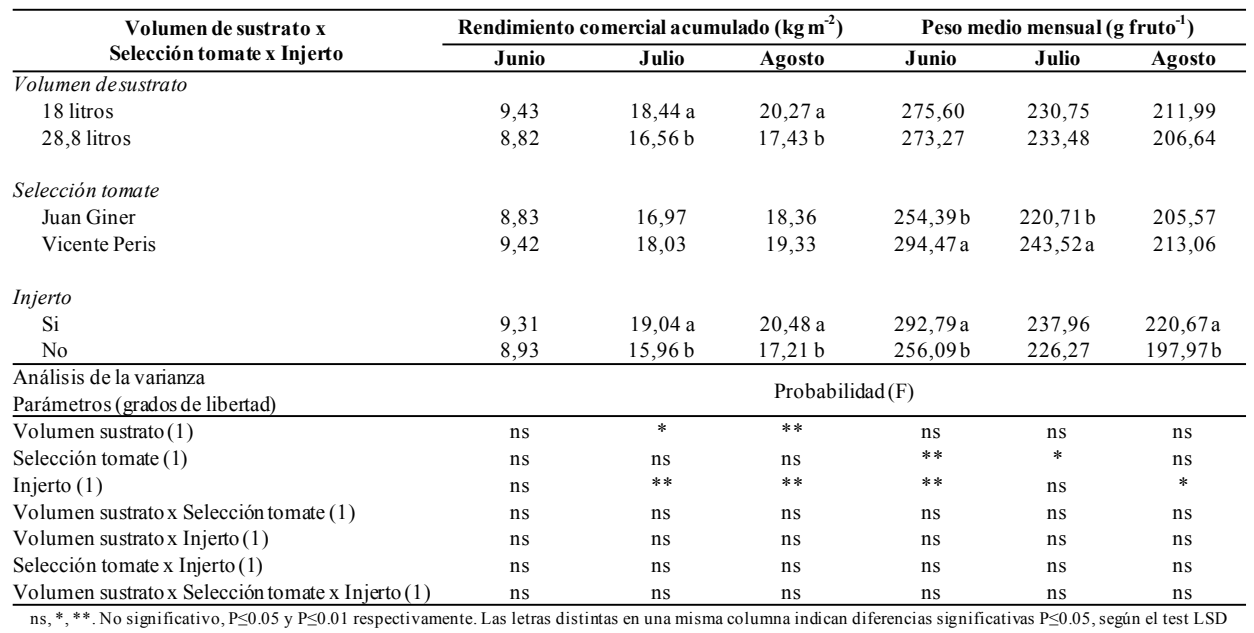


La mayor producción de destrío se obtuvo con los sacos de 18 litros, aunque sin observar d.s.n.e. respecto al saco de mayor volumen. Con el menor volumen de sustrato se detectó una mayor susceptibilidad e.s. a la presencia de frutos con blossom end rot (BER) y no se observaron diferencias entre volúmenes de sustrato para el destrío debido a frutos con agrietado. La selección de Vicente Peris produjo una mayor cantidad de destrío total observando d.s.n.e. Esa mayor cantidad de destrío fue como consecuencia de la mayor susceptibilidad detectada en esta selección al BER, observando d.s.n.e. respecto a la de Juan Giner. Por el contrario, la selección de Juan Giner fue más susceptible al agrietado que la de Vicente Peris, observando d.s.n.e. Las plantas no injertadas dieron una mayor cantidad de destrío total, observando d.s.n.e. respecto a las injertadas, destacó la producción de destrío como consecuencia de BER, en la que se mostró más susceptibles a dicha alteración los frutos de plantas no injertadas, observando d.s.n.e. En la producción de destrío debida a BER, se detectó una interacción e.s. entre volumen de sustrato y selección de tomate, que se explica por el hecho de que para este parámetro no se observaron d.s.n.e. entre sustratos para la selección de Juan Giner, en cambio sí que se observó una mayor susceptibilidad e.s. para el sustrato de 18 litros en la selección de Vicente Peris. Para esta fisiopatía también se observó una interacción e.s. entre selección de tomate y utilización de injerto, que se explica por el hecho de que esa mayor susceptibilidad detectada para las plantas sin injertar fue e.s. para la selección de Vicente Peris, y no se observaron d.s.n.e. en el caso de la selección de Juan Giner. 
Tabla 2. Producción de destrío

\begin{tabular}{|c|c|c|c|}
\hline \multirow{2}{*}{$\begin{array}{c}\text { Volumen de sustrato } x \\
\text { Selección tomate } x \text { Injerto }\end{array}$} & \multicolumn{3}{|c|}{ Destrío $\left(\mathrm{kg} \mathrm{m}^{-2}\right)$} \\
\hline & Blossom end rot & Agrietado & Total \\
\hline \multicolumn{4}{|l|}{ Volumen de sustrato } \\
\hline 18 litros & $2,83 \mathrm{a}$ & 1,69 & 5,96 \\
\hline 28,8 litros & $2,37 \mathrm{~b}$ & 1,69 & 5,33 \\
\hline \multicolumn{4}{|l|}{ Selección tomate } \\
\hline Juan Giner & $0,85 \mathrm{~b}$ & $2,04 \mathrm{a}$ & $4,25 \mathrm{~b}$ \\
\hline Vicente Peris & $4,35 \mathrm{a}$ & $1,34 \mathrm{~b}$ & $7,04 \mathrm{a}$ \\
\hline \multicolumn{4}{|l|}{ Injerto } \\
\hline $\mathrm{Si}$ & $1,91 \mathrm{~b}$ & 1,89 & $5,22 \mathrm{~b}$ \\
\hline No & $3,29 \mathrm{a}$ & 1,49 & $6,07 \mathrm{a}$ \\
\hline Análisis de la varianza & \multirow{2}{*}{\multicolumn{3}{|c|}{ Probabilidad (F) }} \\
\hline Parámetros (grados de libertad) & & & \\
\hline Volumen sustrato (1) & $*$ & $\mathrm{~ns}$ & $\mathrm{~ns}$ \\
\hline Selección tomate (1) & $* *$ & $*$ & ** \\
\hline Injerto (1) & $* *$ & ns & $*$ \\
\hline Volumen sustrato x Selección tomate (1) & $* *$ & ns & ns \\
\hline Volumen sustrato $\mathrm{x}$ Injerto (1) & $\mathrm{ns}$ & $\mathrm{ns}$ & $\mathrm{ns}$ \\
\hline Selección tomate $x$ Injerto (1) & $* *$ & ns & ns \\
\hline Volumen sustrato $\mathrm{x}$ Selección tomate $\mathrm{x}$ Injerto (1) & ns & ns & ns \\
\hline
\end{tabular}

$\mathrm{ns},{ }^{*}, * *$. No significativo, $\mathrm{P} \leq 0.05$ y $\mathrm{P} \leq 0.01$ respectivamente. Las letras distintas en una misma columna indican diferencias significativas $\mathrm{P} \leq 0.05$, según el test LSD

En la apreciación de la altura de las plantas y frondosidad, no se observaron d.s.n.e. entre volúmenes de sustrato. En cambio si se observaron diferencias de altura y frondosidad entre las selecciones, mostrándose la selección de Vicente Peris como más vigorosa. El injerto también indujo un mayor vigor a las plantas respecto a las plantas sin injertar. En la valoración de la altura de las plantas se detectó una interacción e.s. entre selección de tomate y utilización de injerto, que se explica por el hecho de que el injerto indujo un mayor vigor en la selección de Vicente Peris que en la de Juan Giner. 
Tabla 3. Valoración de altura y frondosidad de planta

\begin{tabular}{|c|c|c|}
\hline \multirow[b]{2}{*}{$\begin{array}{c}\text { Volumen de sustrato } x \\
\text { Selección tomate } x \text { Injerto }\end{array}$} & \multicolumn{2}{|c|}{ Fecha: $13 / 6 / 14$} \\
\hline & $\begin{array}{c}\text { Altura planta } \\
(0-5) \\
\end{array}$ & $\begin{array}{c}\text { Frondosidad } \\
(0-5) \\
\end{array}$ \\
\hline \multicolumn{3}{|l|}{ Volumen de sustrato } \\
\hline 18 litros & 4,13 & 3,33 \\
\hline 28,8 litros & 4,17 & 3,38 \\
\hline \multicolumn{3}{|l|}{ Selección tomate } \\
\hline Juan Giner & $3,75 \mathrm{~b}$ & $3,13 \mathrm{~b}$ \\
\hline Vicente Peris & $4,54 \mathrm{a}$ & $3,58 \mathrm{a}$ \\
\hline \multicolumn{3}{|l|}{ Injerto } \\
\hline $\mathrm{Si}$ & $4,50 \mathrm{a}$ & $3,50 \mathrm{a}$ \\
\hline No & $3,79 \mathrm{~b}$ & $3,21 \mathrm{~b}$ \\
\hline Análisis de la varianza & \multirow{2}{*}{\multicolumn{2}{|c|}{ Probabilidad $(\mathrm{F})$}} \\
\hline Parámetros (grados de libertad) & & \\
\hline Volumen sustrato (1) & ns & ns \\
\hline Selección tomate (1) & $* *$ & $* *$ \\
\hline Injerto (1) & $* *$ & $* *$ \\
\hline Volumen sustrato $\mathrm{x}$ Selección tomate (1) & ns & ns \\
\hline Volumen sustrato $\mathrm{x}$ Injerto (1) & ns & ns \\
\hline Selección tomate x Injerto (1) & $* *$ & ns \\
\hline Volumen sustrato x Selección tomate x Injerto (1) & ns & ns \\
\hline
\end{tabular}

\section{4.- DISCUSIÓN}

El menor volumen de sustrato dio una mayor producción comercial total, aunque indujo una mayor susceptibilidad de sus frutos a la presencia de podredumbre apical, especialmente en la selección que se mostró más susceptible a esta alteración.

La selección de Vicente Peris produjo más destrío, fue más susceptible a podredumbre apical y más vigorosa, en cambio la selección de Juan Giner fue más sensible al rajado de los frutos.

Las plantas injertadas favorecieron en general el desarrollo del cultivo dado que produjeron más que aquellas sin injertar, indujeron un mayor peso medio de los frutos y con esta técnica se redujo la producción de 
destrío, especialmente la presencia de podredumbre apical, sobre todo en la selección más susceptible a esta alteración. La planta injertada también mostró un mayor vigor que las no injertadas.

\section{REFERENCIAS BIBLIOGRÁFICAS}

Baixauli, C., Aguilar, J.M. 2002. Cultivo sin suelo de hortalizas, aspectos prácticos y experiencias. Ed. Generalitat Valenciana Consellería de Agriculura, Pesca y Alimentación.

Giner, A., Aguilar, J.M., Baixauli, C., Nájera, I., Núñez, A. Comportamiento agronómico de diferentes portainjertos en cultivo de tomate valenciano. Actas de Horticultura $\mathrm{n}^{\circ} 65$ XIII Jornadas del grupo de horticultura y I jornadas del grupo de alimentación y salud. Logroño 2014. 183-186.

Miguel, A., De La Torre, F., Baixauli, C., Maroto, J.V., Jordá, C., López, M. y García Jiménez, J. (2007). Injerto de Hortalizas. Ministerio de Agricultura, Pesca y Alimentación. 